\title{
The role of nutrition on cognition and brain health in ageing: a targeted approach
}

\author{
Jim M. Monti ${ }^{1 *}$, Christopher J. Moulton ${ }^{1,2}$ and Neal J. Cohen ${ }^{2,3,4,5,6}$ \\ ${ }^{1}$ Abbott Nutrition, Columbus, OH, USA \\ ${ }^{2}$ Center for Nutrition, Learning, and Memory, University of Illinois at Urbana-Champaign, Urbana, IL, USA \\ ${ }^{3}$ Department of Psychology, University of Illinois at Urbana-Champaign, Urbana, IL, USA \\ ${ }^{4}$ Neuroscience Program, University of Illinois at Urbana-Champaign, Urbana, IL, USA \\ ${ }^{5}$ Beckman Institute, Urbana, IL, USA \\ ${ }^{6}$ Interdisciplinary Health Sciences Initiative, University of Illinois at Urbana-Champaign, Urbana, IL, USA
}

\section{Abstract}

Animal experiments and cross-sectional or prospective longitudinal research in human subjects suggest a role for nutrition in cognitive ageing. However, data from randomised controlled trials (RCT) that seek causal evidence for the impact of nutrients on cognitive ageing in humans often produce null results. Given that RCT test hypotheses in a rigorous fashion, one conclusion could be that the positive effects of nutrition on the aged brain observed in other study designs are spurious. On the other hand, it may be that the design of many clinical trials conducted thus far has been less than optimal. In the present review, we offer a blueprint for a more targeted approach to the design of RCT in nutrition, cognition and brain health in ageing that focuses on three key areas. First, the role of nutrition is more suited for the maintenance of health rather than the treatment of disease. Second, given that cognitive functions and brain regions vary in their susceptibility to ageing, those that especially deteriorate in senescence should be focal points in evaluating the efficacy of an intervention. Third, the outcome measures that assess change due to nutrition, especially in the cognitive domain, should not necessarily be the same neuropsychological tests used to assess gross brain damage or major pathological conditions. By addressing these three areas, we expect that clinical trials of nutrition, cognition and brain health in ageing will align more closely with other research in this field, and aid in revealing the true nature of nutrition's impact on the aged brain.

\section{Key words: Nutrition: Ageing: Cognition: Brain health}

\section{Introduction}

A plethora of data across species indicates that nutrition and dietary patterns modulate brain health during ageing. For example, recent reviews and meta-analyses indicate that adherence to the Mediterranean diet, consisting of plant-based foods, olive oil and seafood, reduces the risk of developing Alzheimer's disease $(\mathrm{AD})^{(1-3)}$. Similar neuroprotective effects against cognitive decline are apparent in studies focusing on dietary intake of vitamin $\mathrm{E}$, vitamin $\mathrm{B}_{12}$ and folate, and fish oils rich in $n-3$ fatty acids ${ }^{(4,5)}$. Conversely, high intake of $n-6$ fatty acids coupled with low intake of $n-3$ fatty acids and/or high saturated fat consumption may be associated with pathological $\operatorname{ageing}^{(5,6)}$.

Research in animal models of ageing and $\mathrm{AD}$ has probed the mechanisms that may underlie these effects in humans, and results indicate that a seemingly diverse group of nutrients or foods have beneficial effects on the ageing brain in rodent models. For instance, vitamin $\mathrm{C}$ has been demonstrated to improve memory in rodents, while the anti-inflammatory compound curcumin, found in the spice turmeric, has been shown to increase neurogenesis (the birth of new neurons) in the rodent hippocampus ${ }^{(7,8)}$. Further, a common method in ageing research is to induce or observe naturally the development of pathological formations characteristic of $\mathrm{AD}$, and then investigate how various interventions may mitigate the accumulation of this pathology. The two principal pathologies studied are extra-cellular amyloid- $\beta$ plaques and intracellular neurofibrillary tangles (i.e. 'Alzheimer-like pathology'). Interestingly, a variety of compounds including curcumin, DHA, blueberries and other polyphenols have been documented to ameliorate Alzheimer-like pathology in rodent models ${ }^{(9-12)}$. Additionally, canines fed a diverse diet rich in vitamins $\mathrm{C}$ and $\mathrm{E}, n-3$ fatty acids, L-carnitine, and polyphenols display similar protection from amyloid- $\beta$ pathology and improved cognition in old age ${ }^{(13-15)}$. One possible mechanism accounting for these effects centres on

Abbreviations: AD; Alzheimer's disease; ASL, arterial spin labelling; BOLD, blood oxygen level-dependent; fMRI, functional MRI; MCI, mild cognitive impairment; MMSE, Mini-Mental State Examination; PFC, prefrontal cortex; RCT, randomised controlled trial.

* Corresponding author: Jim M. Monti, fax +1614 727 5270, email James.Monti@abbott.com 
the reduction of oxidative stress via these nutrients and foods, which in turn inhibits Alzheimer-like pathology.

Despite these promising results, randomised controlled trials (RCT) in older humans searching for causal links between nutrients and cognitive function often produce null results $^{(16-25)}$. Large RCT provide the strongest evidence for determining if a particular nutrient has a direct effect on human cognition or brain health, since random assignment to subject groups minimises any confounding effects that may be present in prospective longitudinal or epidemiological studies. Therefore, given these conflicting results across experimental methodologies, it is conceivable that the effects observed in prospective longitudinal or epidemiological studies on nutrition and cognitive ageing, no matter how well-controlled for extraneous effects, reflect confounding factors other than the nutritional variables of interest. An alternative possibility is that previous RCT in this field may have been suboptimally designed to detect the effects of nutrition on the ageing brain. We favour this latter view, and believe there is an opportunity to leverage the results from the important foundational work in this area to inform future studies. The inability to detect positive results in the complex interaction of nutrition and the ageing brain may be due to innumerable factors. For instance, baseline nutritional status may need to be accounted for more thoroughly, as it may be the case that supplementing a nutrient when the majority of the population has optimal status of that nutrient may not be efficacious. Additionally it may be the case that the interaction of certain nutrients, especially from food intake rather than by supplementation may confer the benefits $^{(4)}$. In this review, however, we specifically suggest that the field re-examine its design and implementation of RCT with learnings from the cognitive neuroscience of ageing in order to more fully realise the type of causal evidence sought from RCT on nutrition in cognitive ageing. In order to deliver more successful nutritional RCT in this area, we suggest that careful attention be paid to: (a) the selection of a population of older adults most likely to benefit from a nutritional intervention, with an emphasis that nutrition plays a key role in the maintenance of health rather than treatment of pathological disease; (b) the domains of cognitive neuroscience investigated, with priority given to areas differentially affected by senescence; and (c) the tools chosen to assess these domains, which need not be the same assessment tools used to evaluate diseased populations.

\section{Population selection}

There exists a vast amount of inter-individual variability in cognition among older adults, as a typical sampling of 65-year-old individuals would reveal. For instance, within cognitively healthy individuals, some individuals may still be working productively in their fields and only experiencing infrequent so-called 'senior moments', while others may be retired and could be more forgetful but would be deemed to have normal cognition for his/her age. Yet, there are others who deal with more significant cognitive difficulties. Some individuals may have declines in cognition greater than expected for their age, but have not progressed to dementia, a condition identified as mild cognitive impairment (MCI). Finally, others may already be in the throes of progressive dementia such as $\mathrm{AD}$. This diverse range of cognitive function in older adults presents a quandary in choosing the population(s) in which cognitive and brain health are most likely to benefit from a nutritional intervention. Populations selected for clinical trials on nutrition and cognitive ageing typically include participants from one to three samples: AD patients, MCI patients, or healthy older adults ( $>60$ years old) with no objective cognitive impairment for their age. Here, we wish to emphasise previous contributions ${ }^{(26,27)}$ highlighting that optimal nutrition can maintain and augment cognition in healthy older adults, with an end-goal of reducing the likelihood of developing MCI or $\mathrm{AD}$ and maximising cognition throughout adult life, rather than modifying cognitive diseases of ageing via nutrition.

\section{Alzheimer's disease}

A substantial proportion of large-scale, double-blind RCT have focused on slowing cognitive decline in those afflicted with AD or another dementia, with the results being largely disappointing (for a review, see Otaegui-Arrazola et $a l .{ }^{(28)}$ ). For instance, trials investigating the efficacy of $\mathrm{B}$ vitamins or $n-3$ fatty acids in mild-to-moderate $\mathrm{AD}$ patients found no beneficial effect of these compounds on slowing disease progression, despite ample evidence from non-RCT studies suggesting a role for these nutrients in cognition late in life $\mathrm{f}^{(16,19,22,23,29)}$. One possible reason for the preponderance of null results in clinical trials with $\mathrm{AD}$ patients is that it may be too late to intervene nutritionally when $\mathrm{AD}$ becomes clinically apparent. It is widely appreciated that before even the earliest symptoms of $\mathrm{AD}$ becoming manifest, pathological changes are occurring in the brain which give rise to 'preclinical AD' and 'MCI'. Thus, the disease process of $\mathrm{AD}$ certainly begins years, if not decades, before the emergence of the clinical syndrome of $\mathrm{AD}^{(30)}$. During the preclinical AD and MCI phases, pathology in the form of amyloid- $\beta$ and hyperphosphorylated tau proteins accumulates in the brain, followed by synaptic dysfunction and neuronal injury and loss ${ }^{(30,31)}$. These processes must occur in sufficient magnitude for the development of any clinically relevant cognitive abnormalities. By the time notable cognitive change that is indicative of the mildest stage of AD begins to occur, considerable deleterious changes in the brain have taken place. In two of the studies above, the mean Mini-Mental State Examination (MMSE) score of the participants was approximately 21 out of 30 , such that the average participant in these trials was just outside of the moderate stage of $\mathrm{AD}$; by the time the disease has reached this stage, substantial brain damage has occurred. (The MMSE is a global cognitive screening tool for dementia on a scale of 0-30. Generally, scores of 21-25 are indicative of mild AD, 11-20 moderate AD, and $0-10$ severe $\mathrm{AD}^{(32)}$. However, individuals with scores above 25 can still be diagnosed with $\mathrm{AD}$, especially in the earliest stages of the disorder.)

Given the years- to decades-long head start of $\mathrm{AD}$, implementing a nutritional intervention at the mild-to-moderate stages of $\mathrm{AD}$ in order to affect cognition seems unlikely to succeed. Furthermore, when considering the notion that pharmaceutical 
approaches such as acetylcholinesterase inhibitors offer only palliative effects ${ }^{(33)}$, it is perhaps unsurprising that singlenutrient interventions tend to fail in this population. Therefore, in order to offer a role for nutrition to modify cognition in older adults, researchers must focus on areas of the ageing spectrum where brain function is less impaired. Supporting this notion, Freund-Levi et al. ${ }^{(29)}$ evaluated a range of AD patients with MMSE scores from 15 to 30 . Across the full spectrum of patients, they found no effect of $n-3$ fatty acids on the rate of cognitive decline over 12 months. However, when selectively looking at the most mildly affected patients (MMSE $\geq 28$ ), $n-3$ fatty acid supplementation attenuated cognitive decline in this small sample $^{(29)}$. The fact that Freund-Levi et al. ${ }^{(29)}$ found a positive effect of $n-3$ fatty acids in patients with MMSE scores between 28 and 30, which more likely reflects a cognitive state akin to $\mathrm{MCI}$ rather than $\mathrm{AD}$, underscores the notion that initiating a nutritional intervention before the onset of dementia is more likely to influence cognition in late life. Nonetheless, two other studies that supplemented $n-3$ fatty acids to participants in the same MMSE range did not see beneficial cognitive effects ${ }^{(17,24)}$, furthering the complexity of this area.

\section{Mild cognitive impairment}

Individuals with a diagnosis of MCI display heterogeneous cognitive impairments that are exacerbated for their age. However, these decrements in cognition have not progressed to the level observed in $\mathrm{AD}$ or other dementias. Importantly though, the clinical development of MCI symptoms is preceded by a protracted period of brain damage ${ }^{(30)}$ occurring in the medial temporal lobe (including the hippocampus) as well as portions of the prefrontal cortex (PFC) and parietal lobe. Therefore, these underlying pathological changes that have developed may make successful nutritional intervention at this stage difficult.

There have been some studies indicative of a cognitive benefit via nutritional supplementation in MCI populations. For instance, cognitive impairment was attenuated in an MCI population with elevated homocysteine levels when receiving $\mathrm{B}$ vitamin supplementation (vitamins $\mathrm{B}_{6}, \mathrm{~B}_{12}$, folic acid) relative to the control group ${ }^{(34)}$. The focus on homocysteine reduction directly addresses previous findings linking high levels of this compound to dementia and white matter damage in the elderly ${ }^{(35,36)}$. Further, a few smaller-scale studies have observed an improvement in cognition when providing $n-3$ fatty acid supplementation to those with $\mathrm{MCI}^{(37-39)}$, and it will be important for larger trials to replicate these beneficial effects.

Despite these successes, not all vitamin trials in MCI patients report positive results, as van Uffelen et al. ${ }^{(40)}$ did not find an improvement following vitamin B supplementation. One possibility explaining these opposing findings concerns dosage, as vitamin $\mathrm{B}_{12}$ and folic acid/folate in too high of a dose may actually worsen cognitive outcomes ${ }^{(41-43)}$. Similarly administration (in supplement form) of the antioxidant vitamin $\mathrm{E}$ in a doubleblind RCT in MCI patients did not reduce the rate at which these participants converted to $\mathrm{AD}^{(21)}$. These results seem at odds with prospective cohort investigations indicating a protective role of dietary vitamin $\mathrm{E}$ intake and cognitive ageing ${ }^{(44,45)}$.
One explanation for these mixed results may be revealed by an investigation of the changes occurring in the brain that give rise to MCI. Similar to AD but on a lesser scale, in order for the clinical symptoms of MCI to become apparent (at least when due to an AD-like aetiology), substantial brain pathology, along with synapse and neuron dysfunction or loss, must accumulate in a 'preclinical phase', which typically occurs on a timescale of years $^{(30)}$. Thus, any nutritional intervention initiated when MCI is clinically apparent faces a disease process with a years-long head start, thereby decreasing the likelihood of success. It is clear from some of the above studies that this head start is not insurmountable (perhaps unlike AD), but including these patients in RCT investigating nutrition and cognitive ageing may mask the beneficial effects of certain nutrients (for example, vitamin E) on brain health, as the nutrient cannot fully or successfully act on the brain in this diminished state. Moreover, while any intervention that can prevent patients from converting to dementia from MCI is highly valuable, when considering quality-of-life issues and overall public health, it would be even more valuable to find approaches that reduce the risk of a healthy older adult from developing MCI. Though not as impaired as an AD patient, the patient with MCI may lose his/ her ability to work or drive, and he/she also faces an increased risk of developing AD. Therefore, identifying roles for nutrition in cognitive ageing that can lower the risk of developing MCI or $\mathrm{AD}$, rather than attempting to use nutrition to treat these disorders outright, is of paramount importance. By focusing efforts on healthy older adults (and even in middle-aged populations), it may be possible to further achieve this goal.

\section{Healthy older adults}

Healthy older adults without a diagnosis of AD or MCI do not have an objective cognitive impairment for their age, as measured by current neuropsychological tests. Importantly, this does not indicate that they are operating at the same cognitive level as someone 40 years their junior. Ageing research demonstrates that healthy older adults exhibit decline in several areas of cognition relative to younger adults, including episodic and working memory, as well as processing speed. Interestingly, some cognitive aspects, such as semantic knowledge, stay the same or even improve with age ${ }^{(46)}$. Furthermore, advancing age is a major risk factor for the development of MCI or $\mathrm{AD}$, so a healthy older adult at age 70 years may still develop MCI or AD by the age of 75 years. Therefore, despite normal cognition for his/her age, any given healthy older adult still has: (a) room for improvement in certain cognitive domains; and (b) an increasing risk of objective cognitive impairment stemming from MCI, AD or another dementia as he/she increases in age. By intervening to address these two related points, nutrition has great potential to maintain or improve healthy cognitive ageing; however, the research to date paints a murky picture as to whether nutrition has a role in cognition and brain health for healthy older adults.

The tension between prospective longitudinal and epidemiological studies on the one hand, and RCT on the other, is perhaps greatest when considering the available data from healthy older adults. Though not without controversy, numerous 
prospective longitudinal and epidemiological studies indicate that dietary intake of $n$ - 3 fatty acids, various vitamins, flavonoids and/or adherence to a Mediterranean-type diet reduces AD risk and/or improves cognition (for a review, see Otaegui-Arrazola et $\left.a{ }^{(28)}\right)$. Despite this, the preponderance of data from nutritional RCT in healthy older adults tilts towards these nutrients not having a modulatory effect in cognitive ageing.

With regard to RCT indicating positive effects, an enhancement for memory was observed in healthy older adult males undergoing long-term $\beta$-carotene supplementation ${ }^{(47)}$. Another report assessed the effect of 24 weeks of DHA administration in older adults who were free of dementia or MCI, but had subjective complaints about their memory. In this population, there was an improvement in memory for the DHA group compared with those receiving the placebo ${ }^{(48)}$. Also, a study investigating 3-year folic acid supplementation in participants aged 50-70 years with elevated ( $>13 \mu \mathrm{mol} / \mathrm{l}$ ) homocysteine levels found that the folic acid group had better scores on memory and information processing speed tasks relative to the placebo group ${ }^{(49)}$. This study had some unique characteristics that may shed light on the proper population and length of time necessary for a nutrition intervention. The study by Durga et $a l{ }^{(49)}$ included younger participants than many studies on ageing and nutrition, as in most studies the youngest included age is 65 years, with the mean age being much higher. The inclusion of a sample of individuals in their fifties may provide a population in which the negative brain changes accruing from ageing are in a more benign state and thus more responsive to nutritional intervention, thereby allowing for a greater preservation of cognitive function that is in turn more readily detectable on cognitive tests. The supplementation period was also 3 years, which is also longer than most studies. Yet the larger examination of the literature paints a more complex picture. A recent meta-analysis of homocysteinelowering $\mathrm{B}$ vitamin supplementation and cognition in the elderly did not find support for the role of $\mathrm{B}$ vitamins on cognition, even when looking at younger subpopulations and including studies of long duration (up to 8 years ${ }^{(50)}$ ). It should be noted that this meta-analysis did include studies investigating cerebrovascular patients, and the primary variable in many trials was not cognition but rather lowering of homocysteine. Nonetheless, these null results are seemingly reinforced by the lack of an effect found by van der Zwaluw et al. ${ }^{(51)}$, who studied the effect of $B$ vitamin treatment and cognition in older adults with elevated homocysteine. As described below, one explanation for these inconsistent findings centres on the neurocognitive domains studied and the tools used to assess these domains.

A final note that warrants discussion in the study of nutrition and cognition in healthy adults concerns the length of time necessary for intervention studies. Ideally, studies should be long enough to capture natural deterioration in cognitive abilities over time and/or of sufficient duration for nutrition to have a biological effect on the brain to improve cognitive performance. When studying healthy adults, especially if the study population includes individuals closer to middle-aged, multi-year longitudinal studies would be the ideal norm. This must be balanced with the pragmatic realities that work against multi-year studies such as cost, dropout rate, etc. Below we advocate for the use of assessment tools more appropriate for a healthy population in including more challenging cognitive tests that may potentially reveal subtle cognitive change over time. Therefore, this may shorten the length of interventions, though studies that seek to understand the role of nutrition on the trajectory of cognitive decline in healthy adults still need to be of sufficient length for that decline to occur.

\section{Conclusion}

There are certainly roles for nutrition with respect to quality of life for all older adults, including those in the end stages of AD. However, nutrition will have the greatest likelihood of influencing cognition when it operates on a brain not yet affected by disease states such as MCI, AD or another type of dementia. In the cases of these diseases, the extant brain changes will often dwarf any modifying role from a newly established nutritional intervention. Rather, the most promising role of nutrition in cognitive ageing is maintenance of healthy brain function and reducing the risk of these diseases from occurring; consequently, the population in which we should seek to establish efficacy of nutritional intervention in cognitive ageing should be healthy adults.

\section{Specific cognitive domains and brain regions}

As noted above, many large-scale RCT in healthy older adults have not fared better than those in $\mathrm{AD}$ or MCI. RCT studying the effects of B vitamins, vitamin E or DHA in healthy older adults have produced null results with respect to improvements in cognition $^{(17,18,20,24,25,52)}$. There is a multitude of reasons why these and other studies have not found any modulation of cognition due to nutritional intervention, such as nutritional factors relating to absorption, or a lack of consideration for the heterogeneous status of participants' baseline nutrient levels. We will focus on an alternative (but not mutually exclusive) explanation of these null results that concerns the cognitive domains investigated and the possible inadequacy of the tools used to measure them.

A large proportion of the published nutritional interventions in ageing has tested broad cognitive domains rather than taking a targeted approach that focuses on the areas of cognition most affected by ageing; further, insensitive tools have often been used as a means of measuring these broad domains. Particularly problematic are cases where blunt tools that assess global cognition, and are intended for dementia screening (for example, MMSE), are used as outcome measures in healthy older adults (for a review, see Macready et al. ${ }^{(53)}$ ). Healthy older adults are not likely to have any meaningful variance on the MMSE because it is a screening tool intended to detect dementia. Even though the MMSE is out of 30 points, a score indicative of mild dementia is 25 or below, with scores of 26-29 indicating questionable dementia ${ }^{(32)}$; therefore, the range of scores for healthy older adults on the MMSE is restricted, and many will be near ceiling, making the detection of cognitive improvements from nutrition extremely difficult. In addition to 
the insensitivity issues that occur with global measures, a global cognition approach is misaligned with what is known both about the brain regions and cognitive domains that decline most in ageing, which are probably the brain areas to benefit from nutritional approaches.

Just as all older adults do not decline cognitively at equal rates, brain regions and associated cognitive functions also vary with their susceptibility to ageing (for a review, see Jagust ${ }^{(54)}$ ). For instance, primary sensory areas such as the visual cortex show little volume reduction with advanced age, and they are even relatively spared from the pathology of severe $\mathrm{AD}^{(55,56)}$. Similarly, even though most individuals associate ageing with cognitive decline, certain areas of cognition, such as semantic memory or vocabulary knowledge, remain stable or increase across the lifespan ${ }^{(46,57)}$. Given that RCT in cognitive ageing use improvement or maintenance of a brain region or cognitive construct as a primary outcome measure, the regions/constructs most susceptible to ageing should be selected for investigation; regions/constructs less affected by the ageing process are not as likely to show change over time as a result of interventions, and should therefore be de-emphasised in RCT of cognitive ageing and nutrition.

\section{Relational memory and the hippocampus}

One brain structure particularly sensitive to ageing is the hippocampus, a structure located near the middle of the brain, within the medial temporal lobes, that primarily supports aspects of memory function (see below). MRI studies measuring the volume of the hippocampus indicate that it begins to shrink at an accelerated rate during the sixth decade of life ${ }^{(58)}$; this volumetric decline probably reflects, at least in part, loss and alternation of synapses and a reduction in proliferation of neurons, but little overt neuronal death in individuals free of neurodegenerative disorders ${ }^{(59)}$. Additionally, nearly all adults over the age of 65 years, even those without a diagnosis of MCI or $\mathrm{AD}$, will have some degree of $\mathrm{AD}$-like pathology in the form of neurofibrillary tangles within the medial temporal lobe, and these tangles are negatively associated with memory performance ${ }^{(60)}$. When occurring in adults without MCI or AD, this pathology is almost always limited to the transentorhinal and entorhinal cortices, which are located adjacent to, and are densely connected with, the hippocampus; this pathology can also be found in portions of the hippocampus proper in healthy older adults $^{(61)}$.

While the hippocampus contributes to a variety of cognitive abilities, its main function may be to serve as the critical hub for a network underlying relational (associative) memory formation and retrieval. Through its cellular architecture that displays disproportionate and rapid plasticity, and anatomical location, which serves as a convergence zone for cortical afferents, the hippocampus is capable of binding together various pieces of information into lasting representations (for reviews, see Eichenbaum \& Cohen ${ }^{(62)}$, Cohen \& Eichenbaum ${ }^{(63)}$ and Konkel \& Cohen $\left.{ }^{(64)}\right)$. This system allows one to later remember what the person who introduced himself as 'Roger Atwood' looks like, and that you met him when staying at a hotel the first time you visited Santa Fe. Only the hippocampal memory system can learn such novel relations with just one exposure, making it vital to forming new memories of experience throughout a lifetime. Consequently, tests that maximally engage this function, using novel stimuli and novel relations rather than familiar items, are likely to best tap into hippocampal processing.

Given that the hippocampus deteriorates with age, it is unsurprising that relational memory function declines disproportionately as well ${ }^{(65)}$. Relational memory, because it depends upon a network of brain structures interacting with the hippocampus, may be particularly susceptible to ageing and hence particularly useful in the study of nutrition and cognitive ageing. Further, the hippocampus is aided by areas of the PFC and parietal lobe during encoding and retrieval, which serve to modulate functions such as goal-directed behaviour and attention (for reviews, see Cabeza et al. ${ }^{(66)}$, Simons \& Spiers ${ }^{(67)}$ and Wagner et $a l .{ }^{(68)}$ ), and that these structures, too, are sensitive to age-related effects (see below).

Critically, the literature provides reason to believe that these deleterious effects of hippocampal ageing may be partially ameliorated by nutrition. For instance, animal models indicate that intake of flavonoids increases hippocampal neurogenesis, and it is associated with better spatial memory in rodents ${ }^{(10,69,70)}$. Moreover, DHA administration in rodents also has been linked to higher levels of neurogenesis and enhanced hippocampal memory $^{(71,72)}$. In addition to these nutrition-induced changes in gross brain structure that allow for better spatial memory, both DHA and various flavonoids reduce the amount of tau protein deposition in the brain ${ }^{(9,73)}$. Pertinent to hippocampal health in ageing, the neurofibrillary tangles that form in the medial temporal lobe with ageing are composed of hyperphosphorylated tau protein. Though amyloid- $\beta$ has a more widespread distribution in the brain, its accumulation is also known to impair the type of memory processes that rely on the hippocampus. Importantly, evidence exists for potential modulation of amyloid- $\beta$ by diet. In murine models of ageing, DHA and curcumin supplementation reduce the amount of amyloid- $\beta$ burden, with curcumin also preventing amyloid-associated memory impairment ${ }^{(8,10,74)}$. In a canine model of ageing, pleiotropic diets composed of multiple antioxidants can ameliorate amyloid deposition and improve retention of information ${ }^{(14,15)}$. Finally in humans with MCI, more hippocampal tissue was spared over a 2-year period in a group receiving vitamins $\mathrm{B}_{6}, \mathrm{~B}_{12}$ and folic acid supplementation.

\section{Working memory, executive function and the frontoparietal network}

In addition to relational memory, several other cognitive constructs may prove amenable to nutritional intervention due to their reliance on brain networks that are disproportionately affected by ageing processes. One good candidate is working memory, which involves the ability to temporarily maintain information in one's head, especially in the face of interference, and/or to manipulate the temporarily maintained information to achieve a goal. An example of working memory entails recalling the spatial layout and furniture placement of your living room, and then mentally rearranging the furniture to produce a new design. Working memory processes are thought to rely heavily on frontal and parietal regions of the brain ${ }^{(75,76)}$, 
and older adults frequently experience reductions in working memory capabilities ${ }^{(46)}$. A closely related cognitive construct to working memory is executive function, which is even sometimes thought of as a parent category to working memory. Executive function processes entail abilities such as planning, inhibition and fluid reasoning; these abilities largely rely on a fronto-parietal network ${ }^{(77)}$, and it is common to observe agerelated deficits in executive function ${ }^{(78)}$.

Biological mechanisms accounting for these deficits in working memory and executive function centre on age-related changes that occur with the PFC and parietal lobe. Normal ageing is associated with a reduction in synapses in the $\mathrm{PFC}^{(59)}$, and it is additionally known that both the PFC and posterior parietal lobe undergo volume loss with ageing ${ }^{(55)}$. Similar to the hippocampus, intake of $\mathrm{B}$ vitamins has been associated with more grey matter volume ${ }^{(79)}$, suggesting the possibility of $\mathrm{B}$ vitamins to spare cortical grey matter volume, which may in turn have an effect on working memory. Additionally, amyloid- $\beta$ accumulates in a substantial proportion of older adults presumed to be free of $\mathrm{MCI}$ or $\mathrm{AD}$, with particular concentration in the PFC and in areas of the medial parietal lobe, such as the precuneus ${ }^{(80,81)}$. The level of amyloid- $\beta$ in these areas is inversely related to multiple cognitive abilities, including various forms of memory, attention and processing speed ${ }^{(82,83)}$. One intriguing study that manipulated dietary macronutrient proportions over a 4 -week period indicated that a diet low in saturated fat and a low glycaemic index led to reduced levels of amyloid- $\beta$ 42 in the cerebrospinal fluid (a biomarker for amyloid- $\beta$ in the brain) of healthy older adults ${ }^{(84)}$. Future studies are needed to further discern how macronutrient profiles may mediate amyloid- $\beta$ clearance in humans.

\section{Processing speed and white matter}

A major area of decline in ageing is speed of processing, which refers to how quickly one can process information of multiple modalities ${ }^{(46,85)}$. In fact, some have argued that speed of processing may underlie many of the cognitive deficits observed in ageing $^{(86,87)}$. In the brain, processing speed ability is largely dependent on whole-brain white matter integrity ${ }^{(88,89)}$. Normal ageing is also associated with deterioration of the white matter. These changes in the white matter are more pronounced in the $\mathrm{PFC}^{(90)}$. The aetiology of this pathology in the white matter among healthy older adults is most often due to subtle cerebrovascular-related changes, such as arterial stiffening or microscopic bleeding within the brain ${ }^{(54)}$. Moreover, white matter damage is mitigated by $n-3$ fatty acid intake and adherence to a Mediterranean-type $\operatorname{diet}^{(91,92)}$, potentially due to the salutary cardiovascular effects of this type of diet and the relationship between white matter integrity and cerebrovascular integrity.

\section{Conclusion}

To summarise, the cognitive neuroscience of ageing indicates that the hippocampus, fronto-parietal network and white matter disproportionately decline in ageing, and evidence from both animal and human studies suggests a positive role for nutrition on these brain regions, either through the preservation of tissue or reduction of Alzheimer-like pathology. Therefore, the aforementioned cognitive domains may be good targets for nutritional RCT in cognitive ageing, and tests that provide good indices of these networks' structure and function should be emphasised. However, despite the strong reasons for being optimistic, there remain many RCT that have tested for nutritional effects on memory or processing speed with null results. In the remainder of this review, we discuss the need to go beyond identification of appropriate cognitive domains and to consider the design and construction of sufficiently sensitive tools for appropriately assessing those domains in the study of nutritional modulation of cognitive ageing, and discuss how trials can further be informed by sensitive brain imaging techniques.

\section{Assessment tools}

\section{Cognitive measurements}

Through the excellent work of those in the field of neuropsychology, there exists an array of behavioural assessment tools available to measure aspects of cognitive function. Most often, these tests are deployed when evaluating clinical populations, such as those who have sustained a traumatic brain injury or those suspected of having AD. As an example, when measuring hippocampal-dependent memory, the most commonly used tasks involve asking a participant to memorise a list of common words, and then assessing his/her retention for the material after a delay of approximately $20-30 \min ^{(93)}$. In the case of a patient with damage relatively circumscribed to the hippocampus, this type of test (for example, California Verbal Learning Test) works well in detecting the gross memory impairment that follows this injury. Similarly, the current hallmark indication of early clinical $\mathrm{AD}$ is a primary deficit on this type of task with relative preservation in other cognitive domains ${ }^{(94)}$.

Though these tests have worked well in the characterisation of large impairments due to dementia or brain injury, they may not fare as well in detecting the variance in hippocampaldependent memory performance among those within the so-called 'normal range' of cognitive ability. The idea that the classic neuropsychological tests may be insensitive to subtle changes or early pathology is not new, as demonstrated by the classification of individuals with subjective memory impairment. These individuals do not have an objective memory impairment as measured by a delayed-recall test, but they subjectively report, and are often concerned about, their perceived failing memory ability. Interestingly, those who are concerned about the memory lapses they are experiencing are more likely to develop $\mathrm{AD}^{(95)}$. Thus, it is conceivable that these individuals may have preclinical $\mathrm{AD}$, and that their memory deficit is too subtle to be detected by standard measures; nonetheless, the deficit is real and it could indicate the earliest stages of $\mathrm{AD}$.

The issue of subtle, yet meaningful, effects bears directly on the study of nutrition, brain health, and cognitive ageing. It is unlikely that nutritional modulation of brain function will be on the same magnitude as the effects of brain damage; therefore, just as more sensitive tests may be needed to detect the earliest 
stages of $\mathrm{AD}$, there may also be the need to develop sensitive tests to demonstrate the effects of nutrition on the ageing brain. Indeed, it may be the case that some studies have failed to detect a nutritional enhancement in memory, not because nutrition does not benefit memory, but rather that the tool used could not detect the effects of nutrition. To date, the vast majority of RCT assessing cognitive function following nutritional intervention have used standardised neuropsychological tests that may be ill-suited for detecting subtle cognitive change. Below we provide examples of types of tasks often used in cognitive neuroscience research that may be more successful in illustrating an effect of nutrition on cognition in an aged population.

Relational memory assessment: behavioural tasks. Though the types of tasks described above that measure hippocampaldependent memory are not without merit, in the assessment of hippocampal function, researchers in the field of cognitive neuroscience often use tasks where participants must remember the relations of novel stimuli (for example, unfamiliar faces, scenes, or computer-generated images). Such tasks that have proven to be sensitive to hippocampal structure and function include asking the participant to remember novel face-scene pairs ${ }^{(96,97)}$, or the relations among novel, computer-generated images ${ }^{(98)}$. During the 'study phase' in the 'Face-Scene Task', participants are often shown a background novel scene (such as a generic house or beach) on a computer screen for several seconds, followed by a novel face superimposed on the scene for an additional period of time (for example, 2-10 s; study time can be manipulated to vary performance). After exposure to a series of face-scene pairings, the participant then completes the 'test phase', where he or she is asked to indicate if the displayed face was previously shown with the scene, or to choose which of several faces was paired with the background scene. There are many variations of 'foil' or 'lure' trials, which occur when a participant is shown a new face-scene pair they did not see at study; one example of this are 're-pair' trials, where a participant is shown a previously viewed face with a previously viewed scene, but the specific pair did not occur during the study trials (for more detail, see Hannula et al. ${ }^{(96,97)}$, Konkel et al. ${ }^{(98)}$ and Monti et al $\left.{ }^{(99,100)}\right)$. In forcing participants to remember pairings composed of novel materials, the researcher is taking advantage of the singular ability of the hippocampus to bind the new material together into a new relational representation. Further evidence for a hippocampal proclivity for novelty comes from functional MRI (fMRI) studies, which find that the hippocampus is more active to novel stimuli or novel configurations of stimuli, suggesting it plays a role in processing novelty (for a review, see Kumaran \& Maguire $\left.{ }^{(101)}\right)$. Since the effect of nutrition on the hippocampus is likely to be subtle (relative to the effects of brain damage), we suggest that tests assessing relational memory through the use of novel stimuli will maximally engage the hippocampus and raise the likelihood of detecting a change in hippocampal function as a result of nutritional intervention.

An additional consideration in identifying the proper tools for the assessment of nutritional enhancement of relational memory concerns the ability to analyse various conditions and error types within a task. Recently two research groups, independently of each other, have reported a specific error type on a spatial memory task that serves to reveal the nature of hippocampal function. Watson et al. ${ }^{(102)}$ and Pertzov et al. ${ }^{(103)}$ had patients with hippocampal damage perform tests where they had to study and remember the spatial locations of several objects over short time delays (for example, $4 \mathrm{~s}$ ). As an example of this type of test, known as the Spatial Reconstruction Task, participants study the location of several objects (for example, three to five items) on a background such as a blank computer screen; the objective is to remember the location of each object. After the study phase, there is a brief delay where the screen appears blank, followed by the start of the test phase. During the test portion, the objects may appear aligned in a row at the top of the screen, and the participant is asked to use a computer mouse to click-and-drag the objects to where they were presented during the study phase. By not restricting the responses of the participants (i.e. they are free to place the objects anywhere), the data can be analysed in multiple ways. In the studies referenced above, when the experimenters simply assessed how far off in the distance the participant's placed object was to its original location, the effects of hippocampal damage were mild. However, when researchers made their evaluation at the level of relations between object-location bindings, the hippocampal patients performed much worse. Specifically, those with hippocampal lesions made more 'swap' errors in that the locations they chose to place objects were correct, but they chose the wrong objects for those specific locations ${ }^{(102,103)}$. Findings from one condition in Watson et $a l .{ }^{(102)}$ were that patients with hippocampal damage were forty times more likely to make a relational swap error than were the comparison group. A recent study used a version of the task in Watson et al. ${ }^{(102)}$ with novel, computer-generated images as stimuli, and found that the swap error rate in healthy middle-aged and older adults had a numerically higher correlation with hippocampal volume than traditional neuropsychological tests of hippocampal memory, such as delayed recall on word lists ${ }^{(99)}$. For more information on these types of tasks, see Monti et al. ${ }^{(99)}$, Watson et al. ${ }^{(102)}$ and Pertzov et al. ${ }^{(103)}$

The use of open-ended tasks such as those in the spatial reconstruction experiments described above more closely reflects the complex nature of memory. In many memory tests, the outcome measure is all-or-nothing; either the word is recalled or it is not. In actuality, memory is a far more graded phenomenon in that individuals may remember parts of an event and forget other details. Therefore, tasks that only employ binary dependent measures (for example, correct $v$. incorrect) may miss important variance in memory performance. Neuropsychological tasks that incorporate continuous measures of memory, such as response times, along with the binary measures are more likely to capture this variance, as faster responses may be indicative of stronger memory representations (though one must be careful to take baseline response time into account and speed-accuracy trade-offs). Further, the use of confidence judgments (for example, asking individuals how confidently they feel they answered a trial correctly) can also be informative in parsing binary data and capturing individual difference variation on memory paradigms. 
Relational memory assessment: eye tracking. Nearly all memory tasks, including the examples above, ask participants to overtly respond to reveal what information they remember. This may occur in the form of a free recall test where the participant recites as many words as he or she can remember, or in recognition memory tasks where individuals press buttons indicating if the presented stimulus is old or new. However, overtly asking participants about their memory need not be the only way to measure this cognitive ability. An alternative approach is to measure memory via other means, and a useful tool for this has emerged in eye tracking. Briefly, the technique involves a camera recording the position of the eye, which can be used to infer precisely where the participant is looking on a computer display with millisecond temporal resolution. As an example, the Face-Scene Task mentioned above has been developed for use in conjunction with eye tracking. In one version of the task, participants study single face-scene pairs, and are then shown three faces in spatially distinct locations superimposed on a background scene. When one of the faces was previously paired with the displayed scene, participants with intact hippocampi disproportionately direct their eye gaze to that face ${ }^{(97)}$. Memory has been shown to influence viewing patterns in multiple ways, and the eye-tracking technique provides a plethora of potential analyses due to the richness of eye-tracking data (for a review, see Hannula et $\left.a l^{(104)}\right)$. As an example of this measure's sensitivity to subtle change, eye tracking has proven to be able to detect differences in relational memory following an exercise intervention, even though overt behavioural measures failed to detect any change ${ }^{(100)}$. Since it is conceivable that the effects of nutrition on relational memory and the brain are to be a similar order of magnitude as those of exercise (i.e. a smaller effect size than gross brain damage), the use of eye tracking in nutritional interventions may provide a sensitive measure to detect post-intervention change.

Working memory assessment: behavioural tasks. A sharpening of the evaluation of working memory in the context of nutrition, brain health and cognitive ageing may also prove fruitful. The metric of working memory tasks is generally the amount of information one can maintain and manipulate at a given time. One class of tests often used to measure working memory entails the simple presentation of a series of numbers or spatial locations, followed by asking participants to repeat the stimuli in the order they were presented; an example would be the Digit Span Forward test from the Wechsler Adult Intelligence Scale-IV ${ }^{(105)}$. However, given that there is no demand on manipulating or protecting the presented stimuli from interference, these tasks more accurately reflect the simple temporary storage of information, rather than the construct of working memory. Thus, they do not place as large a demand on the fronto-parietal network and are likely to be insensitive to any modulation of brain function via nutrition. Instead, tests that present strings of numbers, letters or other stimuli and ask participants to manipulate them first, and then repeat them (thereby requiring maintenance and manipulation) place greater demand on working memory.
An example of this type of task would entail reading an individual an interleaved list of numbers and letters, and then asking him/her to repeat the letters in alphabetical order, followed by the numbers in numeric order (i.e. Letter-Number Sequencing ${ }^{(105)}$ ). For instance, a participant may be read the sequence: 'L-4-F-2-H-9' whereby the correct response from the participant would be 'F-H-L; 2-4-9'. Thus, the participant must not only retain the information provided, but mentally rearrange it in order to provide the correct response. These measures are often used in clinical settings, but another set of tests exists that is used more often in experimental psychology and cognitive neuroscience. These tests are collectively known as complexspan tasks, and are similar to 'dual-task' paradigms in that a participant must remember information while performing a secondary task, thereby introducing an element of interference to working memory maintenance. For example, in a version of the reading span paradigm (originally reported by Daneman \& Carpenter ${ }^{(106)}$ ), participants read a sentence and are then asked to determine if the sentence makes sense (some sentences do not make semantic sense, such as 'It was raining so I reached for my vacuum'). After making this judgment, the participant is presented with a word (for example, 'tree' or 'phone') and they are to remember this word. This pattern continues for two to ten sentences. At the end of the sequence, the participant is presented with a list of words, some of which were presented during the sequence and some which were not presented. The participant must identify the words they were asked to remember, and can also be asked to put them in the order in which they appeared. Thus, in this task the participant must maintain the single words presented from the interference of reading other words in the sentences and making judgments on them. Other variants of complex-span tasks include performing mathematical operations (operation-span) or spatial information (symmetry-span; for more details on the complex-span tasks, see Conway et al. ${ }^{(107)}$ and Harrison et al. $^{(108)}$ ).

The complex-span tasks and other working memory tests emphasising manipulation plus maintenance are more likely to detect effects of nutrition than simple maintenance paradigms. This is in large part due to the added load placed on the working memory system when one must either remember information in the face of interference, and/or manipulate that information. By increasing the stress on the fronto-parietal network to perform working memory computations, individual differences, such as those due to nutritional status or intervention, are more likely to emerge. Furthermore, many of the complexspan tasks are now computer-based, allowing for the collection of response times along with accuracy, providing additional data with which one can assess the effects of nutrition. Finally, the complex-span tasks also typically have more trials than the Digit Span or Letter-Number sequencing tasks; this allows for a more accurate estimation of one's working memory capacity, since the additional trials reduce noise in the data.

Cognitive assessment tools: summary. There are a few common themes embedded in the discussion of memory tasks potentially more sensitive to nutritional effects on cognition, and these themes can be applied to other realms of cognition 
such as executive function and processing speed. One major idea involves the use of more difficult tasks. Given that the effects of nutrition are likely to be more evident in healthy older adults, the cognitive assessments employed are not meant to be searching for large 'holes' in the brain, but rather, for the subtle variability that characterises cognitive function across even healthy older adults. A second factor involves the collection of more and richer data in cognitive assessment. For instance, one may wish to collect many trials on a task and use average performance to ensure a more representative metric of the cognitive construct that one is measuring, rather than only using the highest number of words recalled on a trial. An even more illuminating approach would be to collect multiple types of data, some of which are continuous in nature (for example, accuracy, response time and eye movements) to more fully characterise an individual's cognitive ability. These approaches are more likely to make evident individual differences in cognitive performance, and may be more sensitive to the effects of nutritional intervention.

As a final note, the psychometric property of reliability warrants mention. Reliability refers to how likely an individual is to receive a similar score on a test if they take it two consecutive times, assuming no major changes in the individual between test administrations. If a test is unreliable, there could be differences in performance between time point zero and one that are not due to differences within the individual. Many of the tests described above are more experimental in nature, and estimates of reliability for many of these tasks do not exist (though this is not true for all). This is in contrast to most clinical neuropsychology tests, where the reliability of a test is often known and generally high. For the purposes of study design and interpreting results, it would be prudent for researchers to follow the lead of neuropsychologists and ascertain reliability estimates for cognitive tests used in nutritional RCT. For further information regarding these issues specifically to testing for effects of foods and nutrients, we refer the reader to de Jager et $a l .{ }^{(109)}$

\section{$M R I$}

The preceding discussion has emphasised the importance of using sensitive cognitive tasks in order to effectively test nutritional enhancement of cognition in the ageing brain; however, a complimentary tool for assessing how nutrition may alter the health of the ageing brain involves using neuroimaging to gather structural and physiological (for example, functional connectivity, blood flow, metabolites) data on the brain. Even in nutritional interventions where there is a null effect on cognitive task data, a finding indicating the sparing of hippocampal tissue as a result of the intervention would surely be regarded as positive in the context of the ageing brain, and this would probably have downstream behavioural effects, even if they cannot be detected by current neuropsychological tests. Below we highlight successful examples of the deployment of MRI to nutrition and cognitive ageing research, and discuss potentially fruitful MRI techniques not yet applied sufficiently in this field. Though this review only covers MRI, we note that there are other neuroimaging techniques such as electroencephalography or near-IR spectroscopy that may be more suitable for certain questions, particularly due to the high cost of MRI. For a full review on neuroimaging techniques and their application to nutrition, we refer the reader to a recent review by Sizonenko et al. ${ }^{(110)}$.

Structural imaging: grey matter volume. As noted in the previous section, synaptic loss and modification as well as lower proliferation of new neurons (in the hippocampus) contribute to age-related declines in brain volume and cortical thickness, with areas such as the hippocampus and PFC more affected than other brain regions. Reliable estimates of the volume of cortical and subcortical structures (for example, the hippocampus) as well as the thickness of the cortex can be obtained from standard high-resolution structural MRI. Crosssectional studies have indicated positive relationships of total grey matter volume in ageing with antioxidant vitamin (B, C, D and E) intake or adherence to a Mediterranean diet ${ }^{(79,91,92)}$. Further, results from a recent longitudinal study indicate that plasma levels of DHA and vitamin D are associated with reduced cortical thinning in prefrontal and parietal regions in ageing $^{(111)}$. Perhaps the best evidence for the modulation of brain atrophy via nutrition comes from intervention studies in MCI patients with elevated homocysteine levels and who consumed supplemental $\mathrm{B}$ vitamins (vitamins $\mathrm{B}_{6}, \mathrm{~B}_{12}$ and folate). In one report, Smith et $a l^{(112)}$ noted that after 2 years of supplementation, the overall brain volume of the $\mathrm{B}$ vitamin group (relative to the placebo group) was higher, indicating reduced atrophy as a result of B vitamin supplementation. In 2013, this group published similar results, using a more targeted approach to show that these effects in those with elevated homocysteine were greatest in the hippocampus and medial temporal lobe cortex, as well as other posterior brain regions ${ }^{(113)}$. This latter report is indicative of the strategy favoured here, where regions particularly susceptible to ageing processes are scrutinised. Finally, and furthering the complexity of this area, a recent report by the same group suggests that the reduction in brain atrophy is partially dependent on plasma $n$ - 3 fatty acid levels, with those having higher levels experiencing less atrophy ${ }^{(114)}$. It is worth mentioning that the results of these $B$ vitamin interventions occurred in MCI patients who are likely to be experiencing a neurodegenerative process; given that neuron loss is not thought to occur in healthy ageing ${ }^{(115,116)}$ the mechanisms through which B vitamins work in the MCI brain may or may not be applicable to the healthy aged brain. Also, structural imaging techniques are best suited for longer interventions (for example, at least 6 months), given that a relatively large amount of change must occur within the brain for detectable differences on standard resolution (for example, $1 \mathrm{~mm}$ isotropic) structural scans. Thus, studies using this technique in shorter interventions may fail to detect any nutritive effect on brain atrophy simply because enough time has not passed.

Structural imaging: white matter integrity. In addition to the reduction in size of certain brain regions that are largely made up of grey matter, ageing is also associated with damage to the white matter. One consistent alteration in white matter 
structure with ageing occurs in the form of "white matter hyperintensities,(117), which are areas in the white matter of high intensity or brightness in magnetic resonance images; these are most apparent on T2-weighted fluid attenuated inversion recovery (FLAIR) images. White matter hyperintensities are heterogeneous in aetiology, but many are due to ischaemic-related changes in the brain; further, they are most pronounced in the frontal white matter, and have been linked to poorer cognition ${ }^{(118-120)}$. Given the relationship between vasculature health and white matter lesions, it stands to reason that nutrition may have a significant modifying effect on white matter hyperintensities, particularly when considering the potential salutary effect of the Mediterranean diet on cardiovascular health ${ }^{(121-123)}$. Consistent with this notion, consumption of fish, as well as serum levels of $n-3$ fatty acids, has been found to be inversely related to white matter hyperintensities ${ }^{(91,124,125)}$. Further research focusing on the relationship between nutrition, vascular health and white matter lesions in ageing may prove fruitful in elucidating the modifying effects of nutrition and brain ageing.

\section{Structural imaging: magnetic resonance spectroscopy. An} underutilised MR technique in the study of nutrition and brain health is magnetic resonance spectroscopy (MRS), which is a non-invasive technique that allows for the quantification of metabolites in the brain, such as choline, creatine and phosphocreatine, and $\mathrm{N}$-acetyl-aspartate. Previous work has indicated that administration of cytidine diphosphate-choline (Citicoline) to older adults increases phosphodiesters in the brain, potentially indicative of increased phospholipid synthesis and turnover; this increase was also correlated with better memory performance ${ }^{(126)}$. Given the unique data afforded by MRS, coupled with reductions in certain metabolite concentrations, such as $\mathrm{N}$-acetyl-aspartate, in normal ageing ${ }^{(127)}$, the inclusion of this technique in imaging protocols may provide important data pertaining to brain metabolite changes in older adults following a nutritional intervention.

Functional imaging: arterial spin labelling. A direct measure of neurovascular health can now be obtained in a non-invasive manner through the use of an MRI technique known as arterial spin labelling ( $\mathrm{ASL}^{(128)}$ ). ASL measures the perfusion of blood through tissue, and can yield a quantifiable and objective measure of the rate of blood flow through the brain. The ASL technique represents a significant advance over other MRI methodologies for measuring blood flow, as it does not involve the injection of radioactive isotopes. ASL sequences now come standard on many 3T MRI machines, and have been featured in widespread clinical and research use (for a review on ASL technology and application, see Detre et al. $\left.{ }^{(129)}\right)$. Though typically used to measure resting cerebral blood flow, ASL can also be implemented in conjunction with cognitive tasks, providing an estimate of brain function as well. Furthermore, ASL can be used to not only obtain whole-brain measures of perfusion, but also region-specific rates of blood flow. The application of regional analyses could allow researchers to investigate specific hypotheses regarding a nutritional intervention's effect on a certain domain of cognition and its putative neural substrate (for example, an intervention may improve relational memory and be correlated with blood flow increases local to the hippocampus; see Heo et al. ${ }^{(130)}$ for such an approach). To the extent that a given nutritional intervention will ameliorate or prevent age-related conditions that reduce cerebral blood flow, such as atherosclerosis, the use of ASL represents a method of untapped potential in investigating nutrition, ageing, and brain health relationships. This technique may be particularly applicable in shorter interventions (less than 6 months) as research from the literature on exercise, ageing and brain health suggests that changes in cerebral blood flow may occur on shorter timescales than changes in gross anatomical structure ${ }^{(131)}$.

Functional imaging: blood oxygen level dependent signal. A more widespread technique implemented in neuroimaging that relies partially on cerebral blood flow is the use of the blood oxygen level-dependent (BOLD) signal in fMRI. Briefly, when a neural event occurs in the brain, there is a shift in the ratio of deoxygenated blood and oxygenated blood, which have different magnetic properties, and this causes a signal increase in the image. Though affected by cerebral blood flow, the BOLD signal is also determined by cerebral blood volume and cerebral oxygen consumption, making it an indirect measure of neural activity ${ }^{(132)}$. Since these changes in blood oxygenation are quite local within the brain, task-related fMRI, where participants perform a cognitive task during fMRI data collection, has been an excellent tool for mapping brain structure to function. Furthermore, it is possible to compare group differences in task-related activity across time, which may be a fruitful technique for nutrition-related interventions of ageing.

One technique that uses the BOLD signal, but does not depend on task-evoked activity to do so, consists of measuring the brain's 'functional connectivity' while at rest. A common method for assessing functional connectivity is to administer a resting-state scan, which simply consists of using a similar MRI sequence for task-based fMRI, but no stimuli are presented and the subject is instructed to lie still. Due to natural fluctuations in the BOLD response, one can conduct an analysis to ascertain which brain areas show synchrony in their fluctuations, and are therefore said to be functionally connected. A remarkably consistent network of brain regions, termed the 'default-mode network', emerges under these conditions (for reviews, see Raichle \& Snyder ${ }^{(133)}$ and Buckner et al $\left.{ }^{(134)}\right)$. This brain network includes the medial parietal cortex and PFC, lateral parietal lobe, and the medial temporal lobe. In ageing, the intrinsic functional connections weaken, leading to a decrease in network coherence, with negative implications for cognition ${ }^{(135)}$. Other networks, such as the fronto-parietal or frontal-executive can also be interrogated with this technique, and there is now research indicating these networks' malleability in response to exercise intervention $^{(136)}$. Thus, an important outcome measure for studies of nutrition and ageing could be functional connectivity, specifically the restoration of functionally connected networks to a more youthful state; to our knowledge this area remains unexplored in the study of nutrition, brain health and cognitive ageing. 
To summarise, a major reason for the failure of RCT to influence cognition via nutrition in older adults may be that the primary tools selected for measuring cognitive performance were not of the appropriate difficulty to detect subtle change. Rather, we expect that valid and reliable tests that maximally engage the neural networks most susceptible to ageing will illuminate the impact of nutrition on cognition. Further, wherever possible, targeted neuroimaging techniques aimed at assessing the structure and physiological function of these networks should be employed to garner an appreciation for the biological effects that nutrition may have on the ageing brain.

\section{Conclusion}

Null results from multiple RCT investigating the cognitive effects of nutritional interventions on ageing may lead to the conclusion that diet does not influence one's cognitive ageing trajectory; however, to draw such an inference would be premature. Rather, a reassessment of strategy and methodology in this field may provide more optimism for detecting nutritional interactions with cognition, particularly in the context of ageing and brain health late in life. We suggest placing a larger emphasis on studying healthy older adults, potentially even those in middle-age, to understand the role of nutrition in the maintenance of brain health and the prevention of disease. Critically, the effects of nutrition and cognition in ageing are more likely to manifest if researchers study cognitive domains and brain regions particularly sensitive to the effects of ageing, and assess these areas with more challenging behavioural tests and cutting-edge imaging techniques. With these advances in experimental methodology, we expect that the differential findings between cross-sectional and RCT studies in human subjects will be somewhat reconciled, and a more truthful picture of nutrition's effects on cognitive ageing and brain health will be established.

\section{Acknowledgements}

The present review was supported by the Center for Nutrition, Learning, and Memory at the University of Illinois at UrbanaChampaign.

J. M. M., C. J. M. and N. J. C. conceived the idea for the paper. J. M. M. wrote the paper; J. M. M., C. J. M. and N. J. C. edited and revised the paper.

J. M. M. and C. J. M. are employees of Abbott Nutrition.

\section{References}

1. Lourida I, Soni M, Thompson-Coon J, et al. (2013) Mediterranean diet, cognitive function, and dementia: a systematic review. Epidemiology 24, 479-489.

2. Singh B, Parsaik AK, Mielke MM, et al. (2014) Association of Mediterranean diet with mild cognitive impairment and Alzheimer's disease: a systematic review and meta-analysis. J Alzheimers Dis 39, 271-282.

3. Van de Rest O, Berendsen AA, Haveman-Nies A, et al. (2015) Dietary patterns, cognitive decline, and dementia: a systematic review. Adv Nutr 6, 154-168.
4. Morris MC (2012) Nutritional determinants of cognitive aging and dementia. Proc Nutr Soc 71, 1-13.

5. Gomez-Pinilla F (2008) Brain foods: the effects of nutrients on brain function. Nat Rev Neurosci 9, 568-578.

6. Morris MC \& Tangney CC (2014) Dietary fat composition and dementia risk. Neurobiol Aging 35, Suppl. 2, S59-S64.

7. Kennard JA \& Harrison FE (2014) Intravenous ascorbate improves spatial memory in middle-aged APP/PSEN1 and wild type mice. Behav Brain Res 264, 34-42.

8. Kim SJ, Son TG, Park HR, et al. (2008) Curcumin stimulates proliferation of embryonic neural progenitor cells and neurogenesis in the adult hippocampus. J Biol Chem 283, 14497-14505.

9. Frautschy SA, Hu W, Kim P, et al. (2001) Phenolic antiinflammatory antioxidant reversal of $\mathrm{A} \beta$-induced cognitive deficits and neuropathology. Neurobiol Aging 22, 993-1005.

10. Green KN, Martinez-Coria H, Khashwji H, et al. (2007) Dietary docosahexaenoic acid and docosapentaenoic acid ameliorate amyloid- $\beta$ and tau pathology via a mechanism involving presenilin 1 levels. J Neurosci 27, 4385-4395.

11. Lim GP, Chu T, Yang F, et al. (2001) The curry spice curcumin reduces oxidative damage and amyloid pathology in an Alzheimer transgenic mouse. J Neurosci 21, 8370-8377.

12. Wang J, Ho L, Zhao W, et al. (2008) Grape-derived polyphenolics prevent $A \beta$ oligomerization and attenuate cognitive deterioration in a mouse model of Alzheimer's disease. J Neurosci 28, 6388-6392.

13. Fahnestock M, Marchese M, Head E, et al. (2012) BDNF increases with behavioral enrichment and an antioxidant diet in the aged dog. Neurobiol Aging 33, 546-554.

14. Milgram NW, Zicker SC, Head E, et al. (2002) Dietary enrichment counteracts age-associated cognitive dysfunction in canines. Neurobiol Aging 23, 737-745.

15. Pop V, Head E, Hill M-A, et al. (2010) Synergistic effects of long-term antioxidant diet and behavioral enrichment on $\beta$-amyloid load and non-amyloidogenic processing in aged canines. J Neurosci 30, 9831-9839.

16. Aisen PS, Schneider LS, Sano M, et al. (2008) High-dose $\mathrm{B}$ vitamin supplementation and cognitive decline in Alzheimer disease: a randomized controlled trial. JAMA 300, 1774-1783.

17. Dangour AD, Allen E, Elbourne D, et al. (2010) Effect of $2-y$ $n$-3 long-chain polyunsaturated fatty acid supplementation on cognitive function in older people : a randomized, doubleblind, controlled trial. Am J Clin Nutr 91, 1725-1732.

18. Ford AH, Flicker L, Alfonso H, et al. (2010) Vitamins $\mathrm{B}_{12}, \mathrm{~B}_{6}$, and folic acid for cognition in older men. Neurology $\mathbf{7 5}$, $1540-1547$

19. Kwok T, Lee J, Law CB, et al. (2011) A randomized placebo controlled trial of homocysteine lowering to reduce cognitive decline in older demented people. Clin Nutr 30, 297-302.

20. McMahon JA, Green TJ, Skeaff CM, et al. (2006) A controlled trial of homocysteine lowering and cognitive performance. N Engl J Med 354, 2764-2772.

21. Petersen RC, Thomas RG, Grundman M, et al. (2005) Vitamin E and donepezil for the treatment of mild cognitive impairment. N Engl J Med 352, 2379-2788.

22. Quinn JF, Raman R, Thomas RG, et al. (2010) Docosahexaenoic acid supplementation and cognitive decline in Alzheimer disease: a randomized trial. JAMA 304, 1903-1911.

23. Sun Y, Lu C-J, Chien K-L, et al. (2007) Efficacy of multivitamin supplementation containing vitamins $\mathrm{B}_{6}$ and $\mathrm{B}_{12}$ and folic acid as adjunctive treatment with a cholinesterase inhibitor in Alzheimer's disease: a 26-week, randomized, double-blind, placebo-controlled study in Taiwanese patients. Clin Ther 29, 2204-2214. 
24. Van de Rest O, Geleijnse JM, Kok FJ, et al. (2008) Effect of fish oil on cognitive performance in older subjects: a randomized, controlled trial. Neurology 71, 430-438.

25. Kang JH, Cook N, Manson J, et al. (2006) A randomized trial of vitamin $\mathrm{E}$ supplementation and cognitive function in women. Arch Intern Med 166, 2462-2468.

26. Benton D (2010) Neurodevelopment and neurodegeneration: are there critical stages for nutritional intervention? Nutr Rev 68, Suppl. 1, S6-S10.

27. De Jager CA \& Kovatcheva A (2010) Summary and discussion: methodologies to assess long-term effects of nutrition on brain function. Nutr Rev 68, Suppl. 1, S53-S58.

28. Otaegui-Arrazola A, Amiano P, Elbusto A, et al. (2014) Diet, cognition, and Alzheimer's disease: food for thought. Eur J Nutr 53, 1-23.

29. Freund-Levi Y, Eriksdotter-Jönhagen $\mathrm{M}$, Cederholm T, et al. (2006) Omega-3 fatty acid treatment in 174 patients with mild to moderate Alzheimer disease: OmegAD Study: a randomized double-blind study. Arch Neurol 63, 1402-1408.

30. Sperling RA, Aisen PS, Beckett L, et al. (2011) Toward defining the preclinical stages of Alzheimer's disease: recommendations from the National Institute on Aging-Alzheimer's Association workgroups on diagnostic guidelines for Alzheimer's disease. Alzheimers Dement 7, 280-292.

31. Jack CR, Knopman DS, Jagust WJ, et al. (2010) Hypothetical model of dynamic biomarkers of the Alzheimer's pathological cascade. Lancet Neurol 9, 119-128.

32. Perneczky R, Wagenpfeil S, Komossa K, et al. (2006) Mapping scores onto stages: Mini-Mental State Examination and clinical dementia rating. Am J Geriatr Psychiatry 14, 139-144.

33. Aisen PS, Cummings J \& Schneider LS (2012) Symptomatic and nonamyloid/tau based pharmacologic treatment for Alzheimer disease. Cold Spring Harb Perspect Med 2, a006395.

34. De Jager CA, Oulhaj A, Jacoby R, et al. (2012) Cognitive and clinical outcomes of homocysteine-lowering B-vitamin treatment in mild cognitive impairment: a randomized controlled trial. Int J Geriatr Psychiatry 27, 592-600.

35. Dufouil C, Alpérovitch A, Ducros V, et al. (2003) Homocysteine, white matter hyperintensities, and cognition in healthy elderly people. Ann Neurol 53, 214-221.

36. Seshadri S, Beiser A, Selhub J, et al. (2002) Plasma homocysteine as a risk factor for dementia and Alzheimer's disease. $N$ Engl J Med 346, 476-483.

37. Chiu C-C, Su K-P, Cheng T-C, et al. (2008) The effects of omega-3 fatty acids monotherapy in Alzheimer's disease and mild cognitive impairment: a preliminary randomized double-blind placebo-controlled study. Prog Neuropsychopharmacol Biol Psychiatry 32, 1538-1544.

38. Kotani S, Sakaguchi E, Warashina S, et al. (2006) Dietary supplementation of arachidonic and docosahexaenoic acids improves cognitive dysfunction. Neurosci Res 56, 159-164.

39. Lee LK, Shahar S, Chin A-V, et al. (2013) Docosahexaenoic acid-concentrated fish oil supplementation in subjects with mild cognitive impairment (MCI): a 12-month randomised, double-blind, placebo-controlled trial. Psychopharmacology (Berl) 225, 605-612.

40. Van Uffelen JGZ, Chinapaw MJM, van Mechelen W, et al. (2008) Walking or vitamin B for cognition in older adults with mild cognitive impairment? A randomised controlled trial. Br J Sports Med 42, 344-351.

41. Eussen SJ, de Groot LC, Joosten LW, et al. (2006) Effect of oral vitamin B-12 with or without folic acid on cognitive function in older people with mild vitamin B-12 deficiency: a randomized, placebo-controlled trial. Am J Clin Nutr 84, 361-370.

42. Kwok T, Lee J, Lam L, et al. (2008) Vitamin $\mathrm{B}_{12}$ supplementation did not improve cognition but reduced delirium in demented patients with vitamin $\mathrm{B}_{12}$ deficiency. Arch Gerontol Geriatr 46, 273-282.

43. Morris MC, Evans DA, Schneider JA, et al. (2006) Dietary folate and vitamins B-12 and B-6 not associated with incident Alzheimer's disease. J Alzheimers Dis 9, 435-443.

44. Engelhart MJ, Geerlings MI, Ruitenberg A, et al. (2002) Dietary intake of antioxidants and risk of Alzheimer disease. JAMA 287, 3223-3229.

45. Morris MC, Evans DA, Bienias JL, et al. (2002) Vitamin E and cognitive decline in older persons. Arch Neurol 59, 1125-1132.

46. Park DC, Lautenschlager G, Hedden T, et al. (2002) Models of visuospatial and verbal memory across the adult life span. Psychol Aging 17, 299-320.

47. Grodstein F, Kang JH, Glynn RJ, et al. (2007) A randomized trial of $\beta$ carotene supplementation and cognitive function in men: the Physicians' Health Study II. 167, 2184-2190.

48. Yurko-Mauro K, McCarthy D, Rom D, et al. (2010) Beneficial effects of docosahexaenoic acid on cognition in age-related cognitive decline. Alzheimers Dement 6, 456-464.

49. Durga J, van Boxtel MPJ, Schouten EG, et al. (2007) Effect of 3 -year folic acid supplementation on cognitive function in older adults in the FACIT trial: a randomised, double blind, controlled trial. Lancet 369, 208-216.

50. Clarke R, Bennett D, Parish S, et al. (2014) Effects of homocysteine lowering with B vitamins on cognitive aging: meta-analysis of 11 trials with cognitive data on 22,000. Am J Clin Nutr 100, 657-666.

51. van der Zwaluw NL, Dhonukshe-Rutten RA, Van Wijngaarden JP, et al. (2014) Results of 2-year vitamin B treatment on cognitive performance: secondary data from an RCT. Neurology $\mathbf{8 3}$, 2158-2166.

52. Andreeva VA, Kesse-Guyot E, Barberger-Gateau P, et al. (2011) Cognitive function after supplementation with $\mathrm{B}$ vitamins and long-chain omega-3 fatty acids: ancillary findings from the SU. FOL.OM3 randomized trial. Am J Clin Nutr 94, 278-286.

53. Macready AL, Butler LT, Kennedy OB, et al. (2010) Cognitive tests used in chronic adult human randomised controlled trial micronutrient and phytochemical intervention studies. Nutr Res Rev 23, 200-229.

54. Jagust W (2013) Vulnerable neural systems and the borderland of brain aging and neurodegeneration. Neuron 77, 219-234.

55. Raz N, Ghisletta P, Rodrigue KM, et al. (2010) Trajectories of brain aging in middle-aged and older adults: regional and individual differences. NeuroImage 51, 501-511.

56. Yankner BA, Lu T \& Loerch P (2008) The aging brain. Annu Rev Pathol 3, 41-66.

57. Nyberg L, Maitland SB, Rönnlund M, et al. (2003) Selective adult age differences in an age-invariant multifactor model of declarative memory. Psychol Aging 18, 149-160.

58. Fjell AM, Westlye LT, Grydeland H, et al. (2013) Critical ages in the life course of the adult brain: nonlinear subcortical aging. Neurobiol Aging 34, 2239-2247.

59. Morrison JH \& Baxter MG (2012) The ageing cortical synapse: hallmarks and implications for cognitive decline. Nat Rev Neurosci 13, 240-250.

60. Bennett DA, Wilson RS, Boyle PA, et al. (2012) Relation of neuropathology to cognition in persons without cognitive impairment. Ann Neurol 72, 599-609.

61. Braak H, Thal DR, Ghebremedhin E, et al. (2011) Stages of the pathologic process in Alzheimer disease: age categories from 1 to 100 years. J Neuropathol Exp Neurol 70, 960-969.

62. Eichenbaum H \& Cohen NJ (2001) From Conditioning to Conscious Recollection: Multiple Memory Systems in the Brain. New York: Oxford University Press.

63. Cohen N \& Eichenbaum HJ (1993) Memory, Amnesia and the Hippocampal System. Cambridge, MA: MIT Press. 
64. Konkel A \& Cohen NJ (2009) Relational memory and the hippocampus: representations and methods. Front Neurosci 3, $166-174$.

65. Naveh-Benjamin M (2000) Adult age differences in memory performance: tests of an associative deficit hypothesis. J Exp Psychol Learn Mem Cogn 26, 1170-1187.

66. Cabeza R, Ciaramelli E, Olson IR, et al. (2008) The parietal cortex and episodic memory: an attentional account. Nat Rev Neurosci 9, 613-625.

67. Simons JS \& Spiers HJ (2003) Prefrontal and medial temporal lobe interactions in long-term memory. Nat Rev Neurosci $\mathbf{4}$, 637-648.

68. Wagner AD, Shannon BJ, Kahn I, et al. (2005) Parietal lobe contributions to episodic memory retrieval. Trends Cogn Sci 9, $445-453$.

69. Casadesus G, Shukitt-Hale B, Stellwagen HM, et al. (2004) Modulation of hippocampal plasticity and cognitive behavior by short-term blueberry supplementation in aged rats. Nutr Neurosci 7, 309-316.

70. Van Praag H, Lucero MJ, Yeo GW, et al. (2007) Plant-derived flavanol (-)epicatechin enhances angiogenesis and retention of spatial memory in mice. $J$ Neurosci $\mathbf{2 7}, 5869-5878$.

71. He C, Qu X, Cui L, et al. (2009) Improved spatial learning performance of fat- 1 mice is associated with enhanced neurogenesis and neuritogenesis by docosahexaenoic acid. Proc Natl Acad Sci U S A 106, 11370-11375.

72. Petursdottir AL, Farr SA, Morley JE, et al. (2008) Effect of dietary $n-3$ polyunsaturated fatty acids on brain lipid fatty acid composition, learning ability, and memory of senescence-accelerated mouse. J Gerontol A Biol Sci Med Sci 63, 1153-1160.

73. Wang J, Santa-Maria I, Ho L, et al. (2010) Grape derived polyphenols attenuate tau neuropathology in a mouse model of Alzheimer's disease. J Alzheimers Dis 22, 653-661.

74. Lim GP, Calon F, Morihara T, et al. (2005) A diet enriched with the omega-3 fatty acid docosahexaenoic acid reduces amyloid burden in an aged Alzheimer mouse model. J Neurosci 25, 3032-3040.

75. Gazzaley A \& Nobre AC (2012) Top-down modulation: bridging selective attention and working memory. Trends Cogn Sci 16, 129-135.

76. Kane MJ \& Engle RW (2002) The role of prefrontal cortex in working-memory capacity, executive attention, and general fluid intelligence: an individual-differences perspective. Psychon Bull Rev 9, 637-671.

77. Barbey AK, Colom R, Solomon J, et al. (2012) An integrative architecture for general intelligence and executive function revealed by lesion mapping. Brain 135, 1154-1164.

78. Buckner R (2004) Memory and executive function in aging and $\mathrm{AD}$ : multiple factors that cause decline and reserve factors that compensate. Neuron 44, 195-208.

79. Erickson K, Suever B, Prakash R, et al. (2008) Greater intake of vitamins $\mathrm{B}_{6}$ and $\mathrm{B}_{12}$ spares gray matter in healthy elderly: a voxel-based morphometry study. Brain Res 1553, 20-26.

80. Rodrigue KM, Kennedy KM, Devous MD, et al. (2012) $\beta$-Amyloid burden in healthy aging: regional distribution and cognitive consequences. Neurology 78, 387-395.

81. Sperling RA, Laviolette PS, O'Keefe K, et al. (2009) Amyloid deposition is associated with impaired default network function in older persons without dementia. Neuron 63, 178-188.

82. Kennedy KM, Rodrigue KM, Devous MD, et al. (2012) Effects of $\beta$-amyloid accumulation on neural function during encoding across the adult lifespan. NeuroImage $\mathbf{6 2}, 1-8$.

83. Rentz DM, Amariglio RE, Becker JA, et al. (2011) Face-name associative memory performance is related to amyloid burden in normal elderly. Neuropsychologia 49, 2776-2783.
84. Bayer-Carter JL, Green PS, Montine TJ, et al. (2011) Diet intervention and cerebrospinal fluid biomarkers in amnestic mild cognitive impairment. Arch Neurol 68, 743-752.

85. Salthouse TA (2009) When does age-related cognitive decline begin? Neurobiol Aging 30, 507-514.

86. Salthouse TA (1996) The processing-speed theory of adult age differences in cognition. Psychol Rev 103, 403-428.

87. Salthouse TA (2010) Selective review of cognitive aging. J Int Neuropsychol Soc 16, 754-760.

88. Borghesani PR, Madhyastha TM, Aylward EH, et al. (2013) The association between higher order abilities, processing speed, and age are variably mediated by white matter integrity during typical aging. Neuropsychologia 51, 1435-1444.

89. Wen W, Zhu W, He Y, et al. (2011) Discrete neuroanatomical networks are associated with specific cognitive abilities in old age. J Neurosci 31, 1204-1212.

90. Wen W \& Sachdev P (2004) The topography of white matter hyperintensities on brain MRI in healthy 60- to 64-year-old individuals. NeuroImage 22, 144-154.

91. Bowman GL, Silbert LC, Howieson D, et al. (2012) Nutrient biomarker patterns, cognitive function, and MRI measures of brain aging. Neurology 78, 241-249.

92. Scarmeas N, Luchsinger J, Stern Y, et al. (2011) Mediterranean diet and magnetic resonance imaging-assessed cerebrovascular disease. Ann Neurol 69, 257-268.

93. Lezak MD, Howieson DB, Loring DW, et al. (2004) Neuropsychological Assessment, 4th ed. New York: Oxford University Press.

94. Weintraub S, Wicklund AH \& Salmon DP (2012) The neuropsychological profile of Alzheimer disease. Cold Spring Harb Perspect Med 2, a006171.

95. Jessen F, Wiese B, Bachmann C, et al. (2010) Prediction of dementia by subjective memory impairment: effects of severity and temporal association with cognitive impairment. Arch Gen Psychiatry 67, 414-422.

96. Hannula DE, Tranel D \& Cohen NJ (2006) The long and the short of it: relational memory impairments in amnesia, even at short lags. $J$ Neurosci 26, 8352-8359.

97. Hannula DE, Ryan JD, Tranel D, et al. (2007) Rapid onset relational memory effects are evident in eye movement behavior, but not in hippocampal amnesia. J Cogn Neurosci 19, 1690-1705.

98. Konkel A, Warren DE, Duff MC, et al. (2008) Hippocampal amnesia impairs all manner of relational memory. Front Hum Neurosci $\mathbf{2}, 15$.

99. Monti JM, Cooke GE, Watson PD, et al. (2015) Relating hippocampus to relational memory processing across domains and delays. J Cogn Neurosci 27, 234-245.

100. Monti JM, Hillman CH \& Cohen NJ (2012) Aerobic fitness enhances relational memory in preadolescent children: the FITKids randomized control trial. Hippocampus 22, 1876-1882.

101. Kumaran D \& Maguire EA (2009) Novelty signals: a window into hippocampal information processing. Trends $\operatorname{Cogn} \mathrm{Sci}$ 13, 47-54.

102. Watson PD, Voss JL, Warren DE, et al. (2013) Spatial reconstruction by patients with hippocampal damage is dominated by relational memory errors. Hippocampus $\mathbf{2 3}$, 570-580.

103. Pertzov Y, Miller TD, Gorgoraptis N, et al. (2013) Binding deficits in memory following medial temporal lobe damage in patients with voltage-gated potassium channel complex antibody-associated limbic encephalitis. Brain 136, 2474-2485.

104. Hannula DE, Althoff RR, Warren DE, et al. (2010) Worth a glance: using eye movements to investigate the cognitive neuroscience of memory. Front Hum Neurosci 4, 166. 
105. Wechsler D (2008) Wechsler Adult Intelligence Scale, 4th ed. San Antonio, TX: Pearson.

106. Daneman M \& Carpenter PA (1980) Individual differences in working memory and reading. J Verbal Learn Verbal Behav 19, 450-466.

107. Conway AR, Kane MJ, Bunting MF, et al. (2005) Working memory span tasks: a methodological review and user's guide. Psychonom Bull Rev 12, 769-786.

108. Harrison TL, Shipstead Z, Hicks KL, et al. (2013) Working memory training may increase working memory capacity but not fluid intelligence. Psychol Sci 24, 2409-2419.

109. de Jager CA, Dye L, de Bruin EA, et al. (2014) Criteria for validation and selection of cognitive tests for investigating the effects of foods and nutrients. Nutr Rev 72, 162-179.

110. Sizonenko SV, Babiloni C, Sijben JW, et al. (2013) Brain imaging and human nutrition: which measures to use in intervention studies? Adv Nutr 4, 554-556.

111. Walhovd KB, Storsve AB, Westlye LT, et al. (2014) Blood markers of fatty acids and vitamin $\mathrm{D}$, cardiovascular measures, body mass index, and physical activity relate to longitudinal cortical thinning in normal aging. Neurobiol Aging 35, 1055-1064.

112. Smith AD, Smith SM, de Jager CA, et al. (2010) Homocysteine-lowering by $\mathrm{B}$ vitamins slows the rate of accelerated brain atrophy in mild cognitive impairment: a randomized controlled trial. PLOS ONE 5, e12244.

113. Douaud G, Refsum H, de Jager CA, et al. (2013) Preventing Alzheimer's disease-related gray matter atrophy by B-vitamin treatment. Proc Natl Acad Sci US A 110, 9523-9528.

114. Jernerén F, Elshorbagy AK, Oulhaj A, et al. (2015) Brain atrophy in cognitively impaired elderly: the importance of long-chain $\omega$-3 fatty acids and $B$ vitamin status in a randomized controlled trial. Am J Clin Nutr 102, 215-221.

115. Peters A, Morrison JH, Rosene DL, et al. (1998) Feature article: are neurons lost from the primate cerebral cortex during normal aging? Cereb Cortex 8, 295-300.

116. Rapp PR \& Gallagher M (1996) Preserved neuron number in the hippocampus of aged rats with spatial learning deficits. Proc Natl Acad Sci U S A 93, 9926-9930.

117. Gunning-Dixon FM, Brickman AM, Cheng JC, et al. (2009) Aging of cerebral white matter: a review of MRI findings. Int J Geriatr Psychiatry 24, 109-117.

118. Gunning-Dixon FM \& Raz N (2000) The cognitive correlates of white matter abnormalities in normal aging: a quantitative review. Neuropsychology 14, 224-232.

119. Sachdev P, Wen W, Chen X, et al. (2007) Progression of white matter hyperintensities in elderly individuals over 3 years. Neurology $\mathbf{6 8}, 214-222$.

120. Schmidt R, Schmidt H, Haybaeck J, et al. (2011) Heterogeneity in age-related white matter changes. Acta Neuropathol 122, $171-185$.
121. Estruch R, Ros E, Salas-Salvadó J, et al. (2013) Primary prevention of cardiovascular disease with a Mediterranean diet. N Engl J Med 368, 1279-1290.

122. Rees K, Hartley L, Flowers N, et al. (2013) "Mediterranean" dietary pattern for the primary prevention of cardiovascular disease. The Cochrane Database of Systematic Reviews 2013, issue 8, CD009825. http://www.mrw.interscience.wiley.com/ cochrane/clsysrev/articles/CD009825/frame.html

123. Gardener H, Scarmeas N, Gu Y, et al. (2012) Mediterranean diet and white matter hyperintensity volume in Northern Manhattan Study. Arch Neurol 69, 251-256.

124. Virtanen JK, Siscovick DS, Longstreth WT, et al. (2008) Fish consumption and risk of subclinical brain abnormalities on MRI in older adults. Neurology 71, 439-446.

125. Virtanen JK, Siscovick DS, Lemaitre RN, et al. (2013) Circulating omega-3 polyunsaturated fatty acids and subclinical brain abnormalities on MRI in older adults: the Cardiovascular Health Study. J Am Heart Assoc 2, e000305.

126. Babb SM, Wald LL, Cohen BM, et al. (2002) Chronic citicoline increases phosphodiesters in the brains of healthy older subjects: an in vivo phosphorus magnetic resonance spectroscopy study. Psychopharmacology (Berl) 161, 248-254.

127. Minati L, Grisoli M \& Bruzzone MG (2007) MR spectroscopy, functional MRI, and diffusion-tensor imaging in the aging brain: a conceptual review. J Geriatr Psychiatry Neurol 20, $3-21$.

128. Detre JA, Leigh JS, Williams DS, et al. (1992) Perfusion imaging. Magn Reson Med 23, 37-45.

129. Detre J, Rao H \& Wang D (2012) Applications of arterial spin labeled MRI in the brain. J Mag Reson Imaging 35, 1026-1037.

130. Heo S, Prakash RS, Voss MW, et al. (2010) Resting hippocampal blood flow, spatial memory and aging. Brain Res 1315, 119-127.

131. Chapman SB, Aslan S, Spence JS, et al. (2013) Shorter term aerobic exercise improves brain, cognition, and cardiovascular fitness in aging. Front Aging Neurosci 5, 75.

132. Buxton R (2002) Introduction to Functional Magnetic Resonance Imaging: Principles and Techniques. New York: Cambridge University Press.

133. Raichle ME \& Snyder AZ (2007) A default mode of brain function: a brief history of an evolving idea. NeuroImage $\mathbf{3 7}$, 1083-1090.

134. Buckner RL, Andrews-Hanna JR \& Schacter DL (2008) The brain's default network: anatomy, function, and relevance to disease. Ann N Y Acad Sci 1124, 1-38.

135. Andrews-Hanna JR, Snyder AZ, Vincent JL, et al. (2007) Disruption of large-scale brain systems in advanced aging. Neuron 56, 924-935.

136. Voss MW, Prakash RS, Erickson KI, et al. (2010) Plasticity of brain networks in a randomized intervention trial of exercise training in older adults. Front Aging Neurosci 2, 32. 